\title{
Referenciação à Consulta de Pediatria pelos Cuidados de Saúde Primários
}

\author{
The Referral to Pediatrics Consultation from Primary Health Care
}

Cátia Oliveira Matos*, Joana Santos ${ }^{* *}$, Sílvia Sacramento***, Hugo Braga Tavares ${ }^{* * * *}$, Rosa Arménia Campos ${ }^{* * * *}$

\section{Resumo}

Introdução: Uma das competências nucleares do médico de família é a "Gestão em Cuidados de Saúde Primários” e o processo de referenciação surge como uma ferramenta importante.

Objectivo: Avaliar a qualidade da referenciação do médico de família à consulta externa de Pediatria do Centro Hospitalar de Vila Nova de Gaia/Espinho.

Métodos: Estudo transversal e descritivo, realizado entre 2/2013 e 10/2013. População: indivíduos <18 anos referenciados à consulta de Pediatria através do programa $A L E R T^{\circledR} \mathrm{P} 1$, com primeira consulta marcada entre 01/05 e 30/09/2013. Fonte de dados: pedido de referenciação e questionário de avaliação da qualidade da referenciação preenchido pelos pediatras. Avaliámos vários critérios de qualidade e analisámos os dados através de estatística descritiva.

Resultados: Obtivemos 148 avaliações válidas de pedidos de referenciação de um total de 420 primeiras consultas efetivadas no período de estudo (adesão de 36,4\%). A população tinha em média 7,7 anos e 52,0\% eram do género masculino. As áreas com mais pedidos avaliados foram Imunoalergologia $(25,7 \%)$ e Neurodesenvolvimento (15,5\%). A qualidade global foi considerada aceitável em 57,0\%; a informação clínica pertinente em $83,0 \%$ e a terminologia adequada em 87,0\%. A concordância entre prioridades atribuídas pelo médico de família e triador foi de 92,6\%. Houve concordância maioritária quanto à necessidade de pedido de exames complementares de diagnóstico.

\begin{abstract}
Introduction: One of the major Family Practitioner skills is "Management in Primary Health Care", where the referral process becomes an important tool.
\end{abstract}

Objective: To assess the current quality of Referral to Pediatrics Consultation from the Family Practitioner.

Methods: Transversal and descriptive study, since february/2013 to october/2013. Population: individuals $<18$ years old referred to pediatrics consultation by $A L E R T^{\circledR}$ P1 program and with an appointment between may $1^{\text {st }}$ and september $30^{\text {th }}$ of 2013 . Source of data: Referral request and questionnaire for referral's scientific and technical quality assessment completed by pediatricians. We evaluated several quality criteria and analyzed them using descriptive statistic.

Results: We obtained 148 valid referral evaluations from a total of 420 appointments effectuated in the study period (36,4\% of adhesion). The average age was 7,7 years old and 52,0\% were boys. The areas with more referral evaluations were Allergology $(25,7 \%)$ and Neurodevelopment (15,5\%). The global quality was considered acceptable in 57,0\%; the information quality appropriate in $83,0 \%$ and the terminology adequate in $87,0 \%$. The degree of agreement between the Family Practitioner and the triage maker was $92,6 \%$. There was an agreement on the need for complementary tests in the majority of cases.

\footnotetext{
* Médica na USF Espinho.

** Médica na USF Barão do Corvo

**** Médica na USF Leça

${ }^{* * * *}$ Especialista em Pediatria, Centro Hospitalar Vila Nova de Gaia-Espinho.
} 
Discussão: 0 processo de referenciação é importante e complexo, devendo traduzir uma eficaz articulação entre os Cuidados de Saúde Primários e os Secundários. Continua a ser necessário criar protocolos de referenciação, com uniformização de critérios de prioridade e definição das situações que merecem algum estudo complementar prévio.

Palavras-Chave: Referenciação externa, Cuidados de Saúde Primários, Pediatria.

\section{Introdução}

A última definição de Medicina Geral e Familiar (MGF) foi lançada em 2002 pela Wonca Europa (Sociedade Europeia de MGF) e nela foram enumeradas as competências nucleares do Médico de Família (MF) tidas, portanto, como essenciais à disciplina/especialidade. Uma destas competências é a "Gestão em Cuidados de Saúde Primários”, que pressupõe que o médico de MGF deve ser capaz de: gerir o primeiro contacto com os doentes, tentando cobrir toda a gama de problemas de saúde; coordenar a prestação de cuidados dispensados por outros profissionais de saúde e utilizar os serviços de saúde de modo efetivo e apropriado, assumindo-se como advogado do doente, sempre que necessário ${ }^{1}$. Assim, o processo de referenciação surge como uma ferramenta útil na prática clínica, implicando a partilha de responsabilidade clínica entre profissionais e acarretando consequências diretas na saúde dos doentes e de ordem económica para o sistema de saúde. Os principais objetivos da referenciação, com vista à complementaridade da prestação de cuidados, são o estabelecimento de um diagnóstico, instituição de tratamento médico e/ou cirúrgico ou a realização de determinado meio auxiliar de diagnóstico indisponível nos Cuidados de Saúde Primários (CSP).

Para que este processo se revista de qualidade, determinados parâmetros devem ser assegurados, como: necessidade de referenciação (só se e quando necessário, evitando atrasos); destino mais apropriado (para a especialidade mais apropriada em determinado contexto) e processo de comunicação eficaz (contendo a informação necessária, inteligível e num formato acessível, constando dados sobre a investigação efetuada até ao momento, bem como, objetivos e expectativas do médico e do doente $)^{2}$. Dada a escassez de estudos nacionais a este respeito, especula-se que seja este último elemento o que mais limita a qualidade das referenciações, sendo frequentes as queixas dos profissionais de saúde acerca da má qualidade da informação transmitida.
Discussion: The referral process is important, complex and should translate a good articulation between the Primary and Second Health Care systems. Other corrective measures, such as referral protocols are still being needed, with priority criteria standardized and with definition of situations that deserve some previous investigational study.

Keywords: Referral and consultation, Primary Health Care, Pediatrics.

Garantir, portanto, a qualidade no processo de referenciação é investir na continuidade da prestação de cuidados, utilizando recursos de forma mais eficiente.

Neste contexto, durante a realização do estágio de Pediatria no Centro Hospitalar de Vila Nova de Gaia/Espinho (CHVNG/E), foi detetada uma necessidade comum ao Serviço de Pediatria - a avaliação da qualidade das referenciações efetuadas pelos CSP.

Assim, são objetivos deste trabalho: avaliar a qualidade técnico-científica da referenciação para a consulta externa de Pediatria do CHVNG/E por parte dos CSP através da ferramenta informática $A L E R T^{\circledR} \mathrm{P} 1$, caracterizar a resposta do serviço de Pediatria e a população referenciada.

\section{Métodos}

Realizámos um estudo do tipo transversal e descritivo. A população selecionada foram os utentes com idade inferior a 18 anos referenciados para a consulta externa de Pediatria do CHVNG/E através do programa $A L E R T^{\circledR} \mathrm{P} 1$, com efetivação de consulta entre 1 de maio e 30 de setembro de 2013. Assim, a amostra foi de conveniência.

Realizámos um questionário de avaliação da qualidade técnico-científica da referenciação (Anexo I) destinado a ser preenchido pelo pediatra que realizaria a primeira consulta, no próprio dia. Este questionário foi previamente aprovado em reunião de serviço de Pediatria.

Recolhemos os dados para estudo usando como fonte os questionários preenchidos e os respetivos pedidos de referenciação (PR) que originaram as consultas efetivadas.

Excluímos do estudo as primeiras consultas realizadas não solicitadas através da ferramenta informática 
$A L E R T^{\circledR} \mathrm{P} 1$, bem como, aquelas em que os questionários de avaliação não haviam sido devidamente preenchidos.

Para caracterização da amostra, estudámos as variáveis: idade, género e proveniência do pedido de referenciação.

Para caracterização da resposta, estudámos o tipo de consulta marcada e o tempo decorrido entre a receção do pedido e a marcação da consulta. As restantes variáveis utilizadas para o estudo foram: a concordância da prioridade do PR atribuída pelo médico de família, triador (a nível hospitalar) e pediatra que efetuou a consulta; o conhecimento por parte dos pais ou outros

\begin{tabular}{l} 
Critério de Avaliação \\
Existência de informação dada ao(s) acompanhante(s) \\
sobre o tipo de consulta de referenciação \\
\hline $\begin{array}{l}\text { Existência de informação dada ao adolescente sobre o } \\
\text { tipo de consulta de referenciação }\end{array}$ \\
\hline $\begin{array}{l}\text { Existência de informação dada aos acompanhante(s) } \\
\text { sobre o motivo de referenciação }\end{array}$ \\
Existência de informação dada ao adolescente sobre o \\
motivo de referenciação \\
\hline $\begin{array}{l}\text { Existência de informação clínica adequada ao motivo } \\
\text { do PR }\end{array}$ \\
\hline $\begin{array}{l}\text { Existência de informação clínica com terminologia } \\
\text { adequada }\end{array}$
\end{tabular}

Qualidade global do conteúdo do PR

Adequação de ECD realizados

\begin{tabular}{|c|c|}
\hline & Denominador: $\mathrm{n}^{\circ}$ total de $\mathrm{Q}$ válidos \\
\hline Necessidade de realização de ECD & $\begin{array}{l}\text { Numerador: } \mathrm{n}^{\circ} \text { de “sim” à pergunta } 4 \mathrm{~b} \text { do } \mathrm{Q} \\
\text { Denominador: } \mathrm{n}^{\circ} \text { total de } \mathrm{Q} \text { válidos }\end{array}$ \\
\hline $\begin{array}{l}\text { Adequação da especialidade de Pediatria ao motivo do } \\
\text { pedido }\end{array}$ & $\begin{array}{l}\text { Numerador: } \mathrm{n}^{\circ} \text { de "sim" à pergunta } 5 \text { do } \mathrm{Q} \\
\text { Denominador: } \mathrm{n}^{\circ} \text { total de } \mathrm{Q} \text { válidos }\end{array}$ \\
\hline $\begin{array}{l}\text { Adequação da subespecialidade de consulta ao motivo } \\
\text { do pedido }\end{array}$ & $\begin{array}{l}\text { Numerador: } \mathrm{n}^{\circ} \text { de "sim" à pergunta } 6 \text { do Q } \\
\text { Denominador: } \mathrm{n}^{\circ} \text { total de Q válidos }\end{array}$ \\
\hline $\begin{array}{l}\text { Correspondência entre a prioridade do pedido de } \\
\text { consulta atribuída pelo MF e pelo triador }\end{array}$ & $\begin{array}{l}\text { Numerador: n de PR com atribuição de prioridade } \\
\text { "normal” pelo MF e pelo triador ou prioridade “urgente" } \\
\text { pelo MF e “prioritário" ou “muito prioritário" pelo triador } \\
\text { Denominador: n total de Q válidos }\end{array}$ \\
\hline $\begin{array}{l}\text { Correspondência entre a prioridade do pedido de } \\
\text { consulta atribuída pelo MF e pelo médico que realizou } \\
\text { a consulta }\end{array}$ & $\begin{array}{l}\text { Numerador: } n^{\circ} \text { de PR com atribuição de prioridade } \\
\text { "normal” pelo MF e pelo médico que realizou a consulta } \\
\text { ou prioridade “urgente" pelo MF e "prioritário" ou “muito } \\
\text { prioritário" pelo médico que realizou a consulta } \\
\text { Denominador: n total de Q válidos }\end{array}$ \\
\hline $\begin{array}{l}\text { Correspondência entre a prioridade do pedido de } \\
\text { consulta atribuída pelo triador e pelo médico que } \\
\text { realizou a consulta }\end{array}$ & $\begin{array}{l}\text { Numerador: } n^{\circ} \text { de PR com atribuição de prioridade } \\
\text { “normal”, "prioritário" ou “muito prioritário" pelo triador e } \\
\text { pelo médico que realizou a consultas } \\
\text { Denominador: } \mathrm{n}^{\circ} \text { total de Q válidos }\end{array}$ \\
\hline
\end{tabular}

Legenda: $\mathrm{n}^{\circ}$ - Número; PR - Pedido de Referenciação; Q - Questionário; MF - Médico de Família; ECD - Exame Complementar de Diagnóstico † Adolescente - utente com idade $\geq 10$ anos acompanhantes e dos adolescentes sobre o motivo e o tipo de consulta da referenciação clínica; a adequação da informação clínica ao motivo do PR; a adequação da terminologia usada no PR; a qualidade global da informação clínica (segundo critérios constantes no questionário e adaptados de Arribas VR et $\mathrm{al}^{3}$ ); a necessidade e adequação dos exames complementares de diagnóstico (ECD) pedidos e descritos no PR e a pertinência da referenciação à especialidade e subespecialidade em questão (Tabela 1).

Tabela 1: Critérios de avaliação em estudo relativos ao questionário e forma de os calcular

Cálculo do Indicador (Numerador/Denominador x 100)

Numerador: $\mathrm{n}^{\circ}$ de "sim" à pergunta 1 do $\mathrm{Q}$

Denominador: $\mathrm{n}^{\circ}$ total de $\mathrm{Q}$ válidos

Numerador: $n^{\circ}$ de "sim” à pergunta $1 a$ do $Q$

Denominador: $\mathrm{n}^{\circ}$ de $\mathrm{Q}$ válidos de adolescentes

Numerador: $\mathrm{n}^{\circ}$ de "sim" à pergunta 2 do $\mathrm{Q}$

Denominador: $n^{\circ}$ total de $\mathrm{Q}$ válidos

Numerador: $\mathrm{n}^{\circ}$ de "sim" à pergunta $2 \mathrm{a}$ do $\mathrm{Q}$

Denominador: $\mathrm{n}^{\circ}$ de $\mathrm{Q}$ válidos de adolescentes

Numerador: $n^{\circ}$ de "sim" à pergunta $3 a$ do $Q$

Denominador: $\mathrm{n}^{\circ}$ total de $\mathrm{Q}$ válidos

Numerador: $\mathrm{n}^{\circ}$ de "sim" à pergunta $3 \mathrm{~b}$ do $\mathrm{Q}$

Denominador: $\mathrm{n}^{\circ}$ total de $\mathrm{Q}$ válidos

Numerador: $\mathrm{n}^{\circ}$ de respostas de cada nível de qualidade

expresso na pergunta $3 \mathrm{c}$ do $\mathrm{Q}$

Denominador: $\mathrm{n}^{\circ}$ total de $\mathrm{Q}$ válidos

Numerador: $\mathrm{n}^{\circ}$ de respostas de cada nível de adequação

expresso na pergunta $4 \mathrm{a}$ do $\mathrm{Q}$

Denominador: $n^{\circ}$ total de $\mathrm{Q}$ válidos

Numerador: $\mathrm{n}^{\circ}$ de "sim" à pergunta $4 \mathrm{~b}$ do

Numerador: $\mathrm{n}^{\circ}$ de "sim" à pergunta 5 do $\mathrm{Q}$

ergunta 6 do Q

Numerador: $\mathrm{n}^{\circ}$ de PR com atribuição de prioridade pelo MF e "prioritário" ou "muito prioritário" pelo triado Denominador: $\mathrm{n}^{\circ}$ total de $\mathrm{Q}$ válidos

Numerador: $n^{\circ}$ de PR com atribuição de prioridade prioritário" pelo médico que realizou a consulta Numerador: $\mathrm{n}^{\circ}$ de PR com atribuição de prioridad pelo médico que realizou a consultas 
Para a realização deste estudo, solicitámos parecer à Comissão de Ética do CHVNG/E e autorização ao Presidente do Conselho de Administração da mesma instituição, tendo sido ambos positivos, estando os autores sujeitos a sigilo profissional. Inserimos os dados em base própria sem qualquer elemento identificativo, utilizando apenas a idade e o género no que respeita a variáveis do utente.

A análise foi descritiva, com apresentação dos resultados sob a forma de número absoluto $(n)$, média e/ ou percentagem, recorrendo ao programa informático Microsoft Office Excel ${ }^{\circledR} 2007$.

\section{Resultados}

No período de estudo, foram realizadas 420 primeiras consultas de Pediatria decorrentes de pedidos efetuados através da ferramenta ALERT ${ }^{\circledR} \mathrm{P} 1$ (média de 84/mês). Obtiveram-se 153 avaliações de PR (taxa de adesão: $36,4 \%)$, tendo sido excluídas cinco avaliações por informação insuficiente - sem pedido $A L E R T^{\circledR} \mathrm{P} 1$ em anexo e sem indicação da data de realização da consulta -, não havendo qualquer elemento identificativo para completar os dados em falta. Obtivemos, assim, um total de 148 avaliações de qualidade dos PR para estudo.

Os PR avaliados referiam-se a uma população com idades compreendidas entre 2 meses e 17 anos de idade (média de 7,7 anos) e 52,0\% eram do género masculino (77 rapazes e 71 raparigas). A maioria dos pedidos era proveniente dos Agrupamentos de Centros de Saúde (ACeS) de Gaia (57,0\%) e Espinho/Gaia (41,0\%), sendo os restantes 2,0\% do ACeS Feira/Arouca e Aveiro Norte.

As áreas específicas da Pediatria com maior número de PR avaliados (Gráfico 1) foram a Imunoalergologia Pediátrica $(25,7 \%)$, o Neurodesenvolvimento $(15,5 \%)$ e a Consulta de Adolescentes (12,2\%).
Gráfico 1: Tipo de consulta onde foi efetuada a avaliação do PR (em número absoluto)

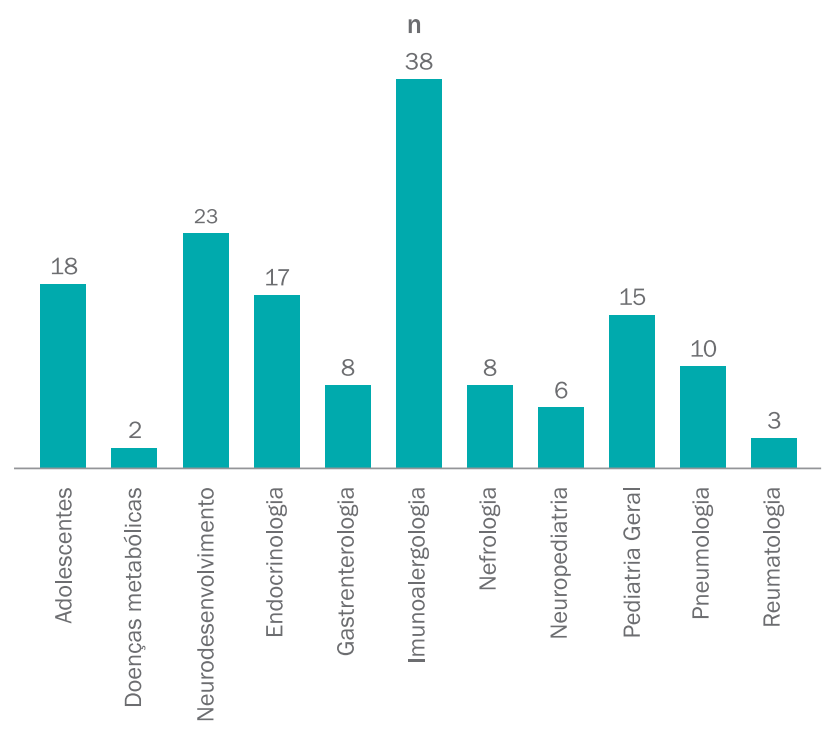

O tempo de demora médio entre o pedido e a marcação da consulta foi de 69,9 dias (mínimo de 14 e máximo de 165 dias).

Relativamente à prioridade do pedido de referenciação efetuado pelo médico de família, 93,0\% foi considerada "Normal" e 7,0\% "Urgente". Comparando com a prioridade atribuída pelo médico triador a nível hospitalar e o médico pediatra que realizou a consulta, observámos uma concordância de $92,6 \%$ e $81,8 \%$, respetivamente (Tabela 2). Por outro lado, a concordância na atribuição de prioridade pelo médico triador e médico pediatra que realizou a consulta foi de $85,1 \%$.

Analisando melhor os casos discordantes entre MF e triador, verificou-se que o triador aumentou a prioridade em 4 casos, todos provenientes de unidades de saúde diferentes, referenciados para subespecialidades também diferentes (Adolescentes, Neurodesenvolvimento, Gastroenterologia e Nefrologia) e com qualidade global do pedido boa (1) ou aceitável (3). Reduziu a prioridade em 7 casos, cujos PR tinham qualidade global aceitável (6) ou má (1).

Como se pode constatar, a discrepância na atribuição de prioridades foi maior quando foi o pediatra a avaliar: dos 21 casos que considerou mais prioritários, apenas 2 foram assinalados como urgentes pelo MF. Por outro lado, dos 10 casos urgentes enviados pelo MF, apenas 3 o eram aos olhos do triador e 2 aos olhos do pediatra. 
Tabela 2: Nível de prioridade atribuído aos pedidos de referenciação pelo médico de família, triador e pediatra (em número absoluto)

\begin{tabular}{|c|c|c|c|}
\hline & \multicolumn{3}{|c|}{ Triagem } \\
\hline Prioridade & Normal & Prioritário & $\begin{array}{c}\text { Muito } \\
\text { Prioritário }\end{array}$ \\
\hline Normal & 134 & 3 & 1 \\
\hline \multirow[t]{2}{*}{ Urgente } & 7 & 3 & - \\
\hline & \multicolumn{3}{|c|}{ Pediatra } \\
\hline Prioridade & Normal & Prioritário & $\begin{array}{c}\text { Muito } \\
\text { Prioritário }\end{array}$ \\
\hline Normal & 119 & 18 & 1 \\
\hline Urgente & 8 & 2 & - \\
\hline
\end{tabular}

Constatou-se que $92,0 \%$ dos pais ou outros acompanhantes estavam informados sobre o tipo de consulta a que a criança tinha sido referenciada (Gráfico 2) e 97,0\% mostraram estar informados sobre o motivo da referenciação.

Relativamente aos adolescentes, 42,0\% disseram saber o tipo de consulta a que foram referenciados e 52,0\% o motivo dessa referenciação (Gráfico 3).

Gráfico 2: Conhecimento dos pais ou outros acompanhantes sobre o tipo de consulta e motivo de referenciação (em percentagem)

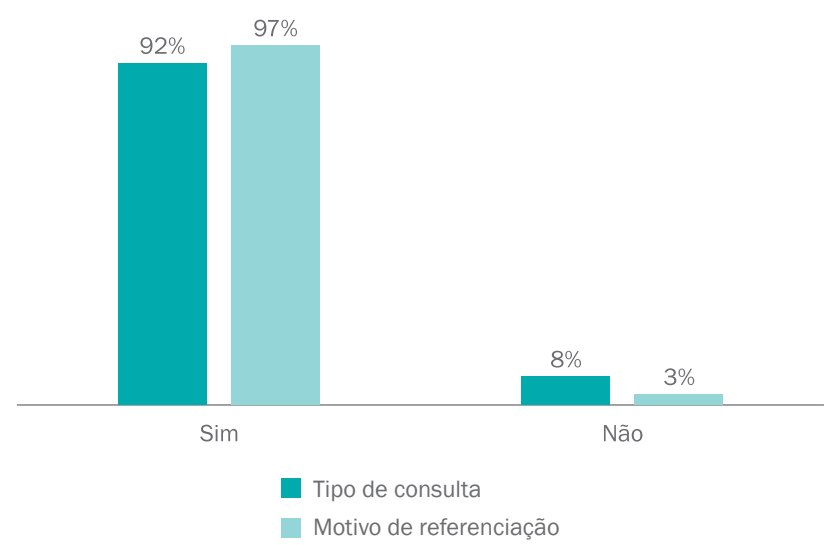

Gráfico 3: Conhecimento dos adolescentes sobre o tipo de consulta e motivo de referenciação (em percentagem)

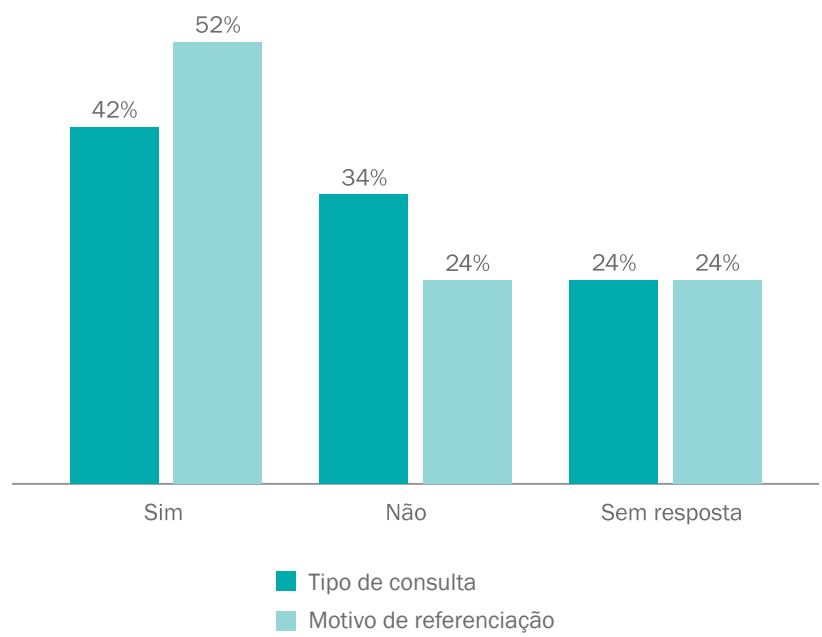

A avaliação da pertinência da informação clínica que constava do PR, tendo em conta o motivo do mesmo, revelou-se adequada em $83,0 \%$ dos casos. Já a terminologia usada foi considerada adequada em 87,0\% dos PR.

A qualidade global dos PR foi considerada pelo pediatra que efetuou a consulta como "Boa" em 29,0\%, "Aceitável" em 57,0\% e "Má" em 14,0\% dos casos.

Vinte e seis por cento dos PR incluíam informação referente a ECD realizados, tendo sido considerados adequados para o motivo de referenciação em $66,0 \%$ dos mesmos (Gráfico 4).

Gráfico 4: Adequação do pedido de Exames Complementares de Diagnóstico ao motivo de referenciação (em percentagem)

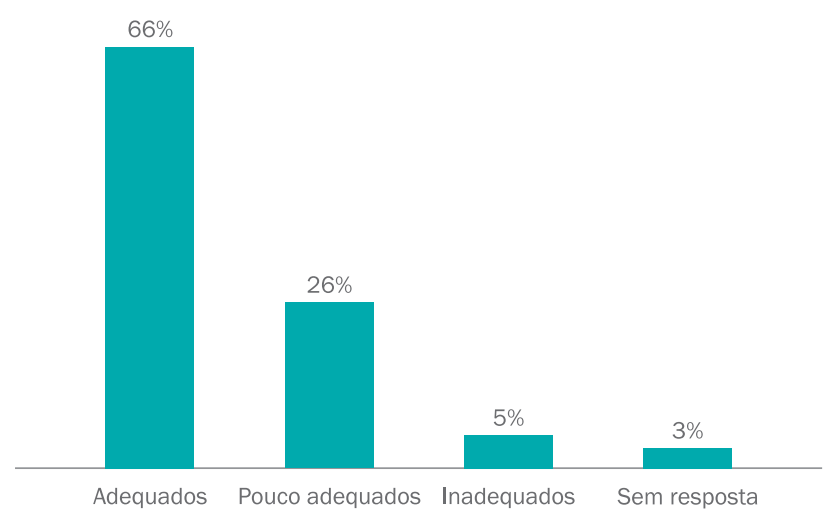


Dos $74,0 \%$ de PR onde não foi descrito o pedido de $E C D$, e interpretando que não haviam sido realizados, os pediatras concordaram que em $87,0 \%$ não havia essa necessidade e que em 11,0\% dos PR havia. Em 2,0\% dos questionários os pediatras não responderam a esta questão.

Relativamente à pertinência da referenciação à especialidade, esta foi considerada adequada em 90,0\% dos casos e não adequada em 1,0\% (9,0\% sem resposta), sendo que em $16,0 \%$ os pediatras avaliadores consideraram haver motivo(s) adicional(is) para referenciação que não estava(m) descrito(s) nos PR.

A pertinência de marcação de consulta por subespecialidade ou área específica da Pediatria foi considerada adequada em $79,0 \%$ dos casos e não adequada em $19,0 \%$, sendo que em 2,0\% não houve resposta.

\section{Discussão}

No período de estudo, cinco meses no total, foram realizadas 420 primeiras consultas decorrentes de PR efetuados através da ferramenta $A L E R T^{\circledR} \mathrm{P} 1$, o que corresponde a uma média de 84 PR por mês.

As áreas com mais PR foram a Imunoalergologia Pediátrica, Neurodesenvolvimentoe Adolescentes.

Foi realizado um estudo em $2011^{4}$, onde avaliaram a qualidade dos pedidos de referenciação à consulta externa de Pediatria efetuados por duas unidades de saúde durante um ano e as áreas com maior referenciação pelos CSP foram a Cirurgia Pediátrica, a Oftalmologia (estes dois não avaliados no nosso estudo) e a Pediatria Geral. Contudo, grande parte (41,5\%) dos motivos de referenciação que foram avaliados nesse estudo foram da área do Neurodesenvolvimento.

Relativamente aos tempos de demora até à realização de primeira consulta, verificámos cumprimento dos tempos médios nos PR considerados "normais" e "prioritários", não tendo sido marginalmente cumprido nos “muito prioritários", segundo o preconizado pela “Consulta a Tempo e Horas" - CTH ${ }^{5}$ (Tabela 3).
Tabela 3: Tempo de espera médio para consulta e comparação com o recomendado pela "Consulta a Tempo e Horas" (número absoluto)

\begin{tabular}{ccc} 
Prioridade triador & $\begin{array}{c}\text { Tempo de } \\
\text { espera máximo } \\
\text { médio (dias) } \\
\text { recomendado } \\
\text { pela } \text { CTH }^{*} \text { (dias) }\end{array}$ \\
\hline Normal & 70,7 & 150 \\
\hline Prioritário & 57,2 & 60 \\
\hline Muito prioritário & 34 & 30
\end{tabular}

${ }^{*} \mathrm{CTH}$ - Consulta a Tempo e Horas

Obtivemos uma boa concordância entre MF e médico triador no que diz respeito à atribuição de prioridade aos PR. Contudo, esta foi inferior entre MF e pediatra que efetuou a consulta e entre este e o médico triador, com maior discordância nos pedidos efetuados e triados com prioridade normal. Esta diferença poderá ser explicada por alguma eventual incongruência entre a informação clínica veiculada no PR efetuado pelo MF, única fonte de informação disponível para o médico triador, e o quadro clínico real, avaliado pelo médico pediatra no momento da consulta. Por outro lado, a própria evolução do quadro clínico durante o tempo de espera até à consulta é um fator a considerar. O que parece evidenciar-se nos casos discordantes é a especificidade de alguns quadros clínicos que poderão suscitar um maior alerta no triador que lê a informação clínica, uma vez que a maioria dos pediatras que avaliou o pedido foi concordante com o triador. Também a qualidade do PR parece ser importante uma vez que apenas um pedido com qualidade "boa" teve a prioridade alterada pelo triador, sendo a avaliação posterior do pediatra que realizou a consulta concordante com a do MF.

Relativamente à informação sobre o tipo de consulta e o motivo de referenciação clínica, verificámos que a maioria dos pais e/ou acompanhantes estavam devidamente informados. No entanto, uma grande parte dos adolescentes desconhecia a que consulta vinha e qual o motivo da mesma, o que poderá ser interpretado como ausência de interesse por parte deles, possível falta de 
maturidade para a perceção dos seus problemas de saúde ou para os conseguirem exprimir e/ou omissão de informação dirigida aos mesmos por parte do MF. De qualquer maneira, este achado realça a importância da comunicação com esta população especial, aliada à necessidade de progressiva responsabilização e autonomia nestas idades. Importa realçar, no entanto, que 24\% dos questionários referentes a adolescentes não tinham registo de resposta a esta questão.

Quanto à qualidade global do PR, foi considerada "Boa" em $29 \%$ dos casos, valor bastante inferior ao obtido no estudo de $2011^{4}$ (69,6\%), onde foram usados critérios idênticos. Consideramos a hipótese de haver uma maior exigência por parte dos pediatras avaliadores, relacionada com o facto de serem diferenciados em áreas mais específicas, o que não aconteceu no estudo de 2011, onde todos os pedidos foram analisados pelos próprios autores. Mesmo assim, 86,0\% dos PR avaliados foram considerados como tendo qualidade global "Boa" ou "Aceitável".

A pertinência quer da informação, quer da terminologia usada nos PR, foi considerada adequada na maioria dos casos, uma vez que o estudo de 2008 não pôde ser incluído na bibliografia.

Quanto aos ECD, verificámos que estes foram realizados em $26,0 \%$ dos casos, sendo que em apenas $5,0 \%$ dos casos a sua realização foi considerada desadequada tendo em conta o motivo do PR. Para além disso, verificámos uma boa concordância (87,0\%) entre o pediatra e o MF nos casos em que não houve pedido de qualquer ECD.

Noventa por cento dos PR foram considerados com pertinência para serem referenciados para a especialidade de Pediatria, sendo que em $16,0 \%$ dos casos foi detetado um motivo adicional que apontava para a pertinência da referenciação que não constava no pedido. Apenas $19,0 \%$ foram considerados pelos pediatras como inadequadamente alocados à subespecialidade ou área específica, sendo este último um critério que se refere à qualidade da triagem realizada no circuito intra-hospitalar, mas a que não é alheia a informação contida no PR que se encontra disponível para o médico triador.
As principais limitações deste trabalho foram a baixa adesão $(36,4 \%)$ no preenchimento dos inquéritos de avaliação por parte dos pediatras que realizaram as primeiras consultas; o período de estudo coincidir com os meses de verão, onde há menor volume de consultas (período habitual de férias); a existência de variabilidade interprofissional nas avaliações (diferentes níveis de exigência, opiniões clínicas, experiências prévias com PR de determinado médico de família e/ou unidade de saúde); existência de perguntas não respondidas por parte dos pediatras e as limitações inerentes ao questionário, idealizado pelos autores do estudo e, portanto, sem validação prévia.

Contudo, consideramos haver pontos fortes neste estudo, como sejam: o número de PR avaliados; o fato dos avaliadores serem especializados em diferentes áreas específicas da Pediatria, introduzindo alta fiabilidade aos resultados, mas também, elevado grau de exigência, e o facto de ser um estudo cego para os MF, que não sabiam que o mesmo estava a decorrer aquando da emissão dos PR.

Em resumo, o processo de referenciação reveste-se de elevada importância e complexidade, devendo traduzir uma eficaz articulação entre os Cuidados de Saúde Primários e Secundários. A informação constante dos PR influencia a prioridade atribuída pelo médico triador $\mathrm{e}$ deverá ser cuidada, completa e pertinente para uma maior qualidade de prestação de cuidados.

Consideramos ser pertinente a existência de protocolos de referenciação claros, com critérios de prioridade bem definidos, descrição das situações que possam merecer realização de ECD prévios com descrição dos mesmos, para que possamos garantir que prestamos o melhor cuidado aos nossos utentes, quer a nível de conhecimento científico como de competências técnicas, capacidade de utilização eficaz e eficiente dos recursos e garantia de acessibilidade.

\section{Agradecimentos}

Aos elementos do serviço de Pediatria do CHVNG/E que colaboraram na investigação e sem os quais este estudo não teria sido possível. 


\section{Referências Bibliográficas}

1. WONCA. A definição europeia de medicina geral e familiar. 2002.

2. Foot $\mathrm{C}$, Naylor $\mathrm{C}$ and Imison $\mathrm{C}$. The quality of GP diagnosis and referral - An Inquiry into the Quality of General Practice in England. The king fund's. 2010.

3. Rubio Arribas $V$ et al Evaluación de la calidad de comunicación entre niveles asistenciales mediante el documento interconsulta. AtenPrimaria. 2000 Dec; 26 (10): 681-4.

4. Santos MI, Coelho I, Rosário F, Machado P, Nery L, Ribeiro J, et al. Referenciação aos cuidados de saúde secundários em idade pediátrica. Rev Port Clin Geral. 2011;27(5):422-32.

5. Ministério da Saúde. Portaria n 95/2013, de 4 de Março de 2013. Diário da República - $1 .^{a}$ série - N. ${ }^{\circ} 44$.

\section{Conflito de Interesses}

Os autores declaram não ter conflitos de interesses.

\section{Endereço para Correspondência:}

Cátia Oliveira Matos

Email: catia.oliveiramatos@gmail.com 
ANEXO I. Questionário

Avaliação dos Pedidos de Consulta de Pediatria

(via ALERT ${ }^{\circledR} \mathrm{P} 1$ )

\section{QUESTIONÁRIO}

Sub-especialidade

Data da consulta: / 2013

1. Os pais (ou outro acompanhante) sabem para que consulta foi feita a referenciação?

$\square \operatorname{Sim}$

$\square$ Não

a) No caso de se tratar de um adolescente, ele sabe a que consulta foi referenciado?

$\square \operatorname{Sim}$

$\square$ Não

2. Os pais (ou outro acompanhante) sabem o motivo pelo qual houve uma referenciação?

$\square \operatorname{Sim}$

$\square$ Não

a) No caso de se tratar de um adolescente, ele sabe o motivo pelo qual foi referenciado?

$\square \operatorname{Sim}$

$\square$ Não

3. Em relação à informação clínica constante no pedido de consulta:

a) Considera-a adequada ao motivo de referenciação?

$\square \operatorname{Sim}$

$\square$ Não

b. Terminologia usada adequadamente?

$\square \operatorname{Sim}$

$\square$ Não

c. Como a caracteriza em termos de qualidade global?

$\square$ Boa

$\square$ Aceitável

$\square$ Má

[ver tabela nas instruções de preenchimento]
4. Foram realizados exames complementares de diagnóstico dirigidos ao motivo de referenciação?

$\square \operatorname{Sim}$

a) Considera-os:

$\square$ Adequados

$\square$ Pouco adequados

$\square$ Inadequados

$\square$ Não

b) Considera que os mesmos eram necessários?

$\square \operatorname{Sim}$

$\square$ Não

5. 0 motivo de consulta é adequado para ser visto por Pediatria? $\square \operatorname{Sim}$

$\square$ Não

a) Existia outro motivo para referenciação que não constasse no pedido?

$\square \operatorname{Sim}$

$\square$ Não

6. 0 motivo de consulta é adequado para ser visto nessa subespecialidade?

$\square \operatorname{Sim}$

$\square$ Não Se não, em qual considera que devia ser visto?

7. A data da $1^{\mathrm{a}}$ consulta foi alterada?

$\square \operatorname{Sim}$ Se sim, $\square$ A pedido do doente

$\square$ A pedido do médico

$\square$ Outro motivo Qual?

Não

8. Como classifica a urgência do pedido?

$\square$ Muito prioritário (30 dias)

$\square$ Prioritário (60 dias)

$\square$ Prioridade normal (150 dias) 
Instruções de preenchimento:

1. Assinalar com uma cruz $(\mathrm{x})$ a resposta que achar mais adequada e, sempre que necessário, escrever a resposta em local próprio para o efeito

2. Para responder à pergunta 3.c), use os seguintes critérios:

Quadro I. Critérios para avaliação da qualidade global do pedido de referenciação. Adaptado de Arribas VR et al ${ }^{3}$

Níveis de Qualidade da Informação clínica contida no pedido de referenciação:

\section{BOA}

Antecedentes pessoais e familiares (se pertinente)

Medicação habitual (se pertinente)

Dados suficientes sobre a doença atual, exame objetivo e exames complementares

Hipótese diagnóstica, diagnóstico diferencial ou dúvidas a esclarecer

\section{ACEITÁVEL}

O motivo de referenciação está bem claro Apresenta dados suficientes sobre a doença atual mas não todos os incluídos na definição anterior de boa qualidade

\section{MÁ}

Incompreensível

Sem dados suficientes para avaliação da doença atual Ausência de informação

\section{No final, anexar o pedido de consulta ao questionário}

Muito grata pela colaboração,

A equipa de investigação. 